\title{
Bioluminescence Resonance Energy Transfer (BRET)-based Biosensing Probes Using Novel Luminescent and Fluorescent Protein Pairs
}

\author{
Hajime Shigeto* $^{*}$ and Shohei Yamamura ${ }^{* *}$ \\ Health Research Institute, National Institute of Advanced Industrial Science and Technology (AIST), \\ 2217-14 Hayashi-cho, Takamatsu, Kagawa 761-0395, Japan
}

(Received July 6, 2018; accepted August 22, 2018)

Keywords: BRET, luminescent protein, fluorescent protein, homogeneous assay, $\mathrm{Ca}^{2+}$ imaging

Bioluminescence resonance energy transfer (BRET) is a useful technique for visualizing cellular functions and responses to stimuli. To construct efficient biosensing protein probes using BRET, novel luminescent and red fluorescent protein pairs, which have separate peaks of luminescence and fluorescence and can cause energy transfer efficiently, were screened. The red fluorescent protein, mScarletI, used as an acceptor, induced a highly efficient BRET signal from green or blue luminescent proteins [Emerald Luc (ELuc) or NanoLuc (NLuc)]. Novel pairs of luminescent and red fluorescent protein (mScarletI) could be applied to the analysis of calcium ions $\left(\mathrm{Ca}^{2+}\right)$. The BRET-based biosensing protein pair of mScarletI and NLuc showed an increased intensity of the BRET signal, depending on the concentration of $\mathrm{Ca}^{2+}(0-4 \mu \mathrm{M})$. Intracellular $\mathrm{Ca}^{2+}$ influx was monitored in HEK293A cells stimulated with $50 \mathrm{mM} \mathrm{KCl}$ and 15 $\mathrm{mM}$ arginine using the BRET-based biosensing protein probe with the novel protein pair. This pair of proteins was particularly suited to cellular imaging in vitro and even in vivo. Therefore, it could be useful for various BRET-based analyses of cell and tissue samples.

\section{Introduction}

Luminescent proteins, such as luciferase, are used in various assay methods designed to evaluate cellular function. ${ }^{(1-3)}$ Bioluminescence resonance energy transfer (BRET) is one of the representative assay methods that uses these luminescent proteins. The BRET-based methods are suitable for homogeneous assays, namely, those without sample collection or a washing step, because the BRET signals are emitted only when the biosensing probe proteins bind with the target molecules. Previously, the BRET-based biosensing probes for detecting insulin $^{(4)}$ and calcium ion $\left(\mathrm{Ca}^{2+}\right)^{(5,6)}$ or measuring intracellular signal transduction ${ }^{(7,8)}$ have been reported. However, most analyses using BRET have utilized a protein pair that includes a blue luminescent protein and a yellow fluorescent protein. This is because the blue luminescent protein and yellow fluorescent protein can increase the BRET signal intensity via highly

\footnotetext{
*Corresponding author: e-mail: hajime.shigeto@aist.go.jp

** Corresponding author: e-mail: yamamura-s@aist.go.jp

https://doi.org/10.18494/SAM.2019.2049
} 
efficient energy transfer. However, the BRET signal generated by these proteins produces a high background and a low signal-to-noise $(\mathrm{S} / \mathrm{N})$ ratio because of the narrow spectrum proximity. ${ }^{(9)}$ In addition, blue and yellow luminescences are unsuitable for analyzing deep tissues in living organisms because the light at these wavelengths has limited permeability in organic tissues. ${ }^{(10)}$ Hence, red fluorescent proteins, which act as the acceptor in BRET signal analysis, having a separate peak wavelength from that of blue or green luminescence, are required for cell and tissue sensing. Although several red fluorescent proteins have been reported, those used in BRET-based assays are limited ${ }^{(8,11)}$ because most of them have lower fluorescence intensities and lower efficiencies of energy transfer. Recently, Chu et al. have reported $^{(12)}$ the orange-red fluorescent protein CyOFP1, which can cause one of the most highly efficient energy transfers using the blue luminescent protein Antares. However, Antares is a fused protein with one luminescent protein and two fluorescent proteins, and this fused protein has a large molecular weight. These large proteins sometimes cause toxicity and may be difficult to express in cells. Therefore, in this study, for the design of small-molecular-weight BRET-based probes, we screened novel pairs of single luminescent and single red fluorescent proteins that can transfer energy efficiently. A novel pair of luminescent and fluorescent proteins was applied to a BRET-based $\mathrm{Ca}^{2+}$-sensing protein probe as a model for a biosensing assay. Furthermore, the $\mathrm{Ca}^{2+}$ influxes into a cultured cell line against stimulations were continuously monitored using the newly developed protein probe.

\section{Materials and Methods}

\subsection{Vector construction}

A plasmid expressing linker-connected luminescent and fluorescent proteins was constructed. First, NanoLuc luciferase ${ }^{(13,14)}$ (NLuc, Promega, Madison, WI, USA), Emerald Luc luciferase $^{(15)}$ (ELuc, Toyobo, Osaka, Japan), and synthesized DNA (Genewiz, South Plainfield, NJ, USA) encoding the mCherry-[Gly-Gly-Gly-Gly-Ser (linker peptide)] or mScarletI ${ }^{(16)}$-[GlyGly-Gly-Gly-Ser (linker peptide)] amino acid sequence were inserted into the pColdII plasmid (Takara Bio, Shiga, Japan). NLuc or ELuc was then inserted at the end of the linker peptide.

To construct the expression plasmid of the $\mathrm{Ca}^{2+}$-sensing protein probe, synthesized DNA (Genewiz) with encoding calmodulin (CaM)-(linker peptide)-the CaM-binding peptide of myosin light-chain kinase (M13) ${ }^{(17-19)}$ was inserted at the end of the NLuc-encoding gene at the pColdII plasmid. Then, the mScarletI-encoding gene was inserted at the end of the M13 encoding sequence. To produce a plasmid to express the $\mathrm{Ca}^{2+}$-sensing protein probe in cellular cytosol, the gene encoding (NLuc)-(CaM)-(linker peptide)-(M13)-(mScarletI) was transferred into the pcDNA3 plasmid (Thermo Fisher Scientific, Waltham, MA, USA).

\subsection{Expression and purification of fused proteins}

The fused fluorescent and luminescent proteins and the $\mathrm{Ca}^{2+}$-sensing protein were expressed using a pCold expression system (Takara Bio) following the manufacturer's protocol with 
BL21(DE3)pLysS (Thermo Fisher Scientific). The expression of each protein was confirmed by sodium dodecyl sulfate-polyacrylamide gel electrophoresis (SDS-PAGE) analysis and purified using His-tag affinity chromatography (data not shown). First, the homogenized bacterial suspension was dialyzed with a His-tag affinity buffer $\left(20 \mathrm{mM} \mathrm{NaH}_{2} \mathrm{PO}_{4} ; 500 \mathrm{mM} \mathrm{NaCl} ; 5\right.$ $\mathrm{mM}$ imidazole; $\mathrm{pH}$ 7.4). The dialyzed sample was added to a Ni-NTA agarose solution (Qiagen, Hilden, Germany), and the target proteins were eluted using an imidazole gradient. The eluted proteins were dialyzed with phosphate-buffered saline (PBS). The purification of the proteins was confirmed by SDS-PAGE analysis (data not shown). The proteins were stored at $4{ }^{\circ} \mathrm{C}$ with $1 \mathrm{mg} / \mathrm{mL}$ bovine serum albumin (BSA, F-V) (Nacalai Tesque, Kyoto, Japan) after the concentration was determined using a Micro BCA Kit (Thermo Fisher Scientific).

\subsection{Functional analysis of fused proteins}

The BRET analysis results of expressed and purified fused luminescent and fluorescent proteins were confirmed by the evaluation of luminescence and fluorescence. Using mCherryNLuc- or mScarletI-NLuc-fused proteins, we measured the luminescence spectra of the protein samples in a volume of $49 \mu \mathrm{L}$ containing $50 \mathrm{nM}$ protein as the final concentration with a CCD spectrometer (Lumi Fluoro Capture, ATTO, Tokyo, Japan) set with an integration time of $5 \mathrm{~s}$ after the addition of $1 \mu \mathrm{L}$ of Nano-Glo Luciferase Assay Substrate (Promega). Using mCherryELuc- or mScarletI-ELuc-fused proteins, we also measured the luminescence spectra of the proteins samples in a volume of $50 \mu \mathrm{L}$ containing $500 \mathrm{nM}$ protein using a CCD spectrometer set with an integration time of $30 \mathrm{~s}$ after the addition of $50 \mu \mathrm{L}$ of Emerald Luc Luciferase Assay Reagent (Toyobo). The BRET efficiency was calculated using the formula emission intensity from acceptor/emission intensity from the donor, with the following characteristics: acceptor emission at $598 \mathrm{~nm}$ (mScarletI) and $610 \mathrm{~nm}$ (mCherry); donor emission at $460 \mathrm{~nm}$ (NLuc) and $538 \mathrm{~nm}$ (ELuc).

For the functional analysis of the protein probe for sensing $\mathrm{Ca}^{2+}$, the BRET signals were measured using the luminescence intensities at 460 and $598 \mathrm{~nm}$ with an integration time of $10 \mathrm{~s}$ using a $5 \mathrm{nM}$ protein probe after the addition of $1 \mu \mathrm{L}$ of Nano-Glo Luciferase Assay Substrate in a total volume of $50 \mu \mathrm{L}$ containing $50 \mathrm{mM}$ etylenediaminetetraacetic acid (EDTA), $1 \mathrm{mg} / \mathrm{mL}$ BSA, and each concentration of $\mathrm{CaCl}_{2}$. The BRET efficiency was calculated using the emission at 598 and $460 \mathrm{~nm}$. All experiments were conducted in PBS with $1 \mathrm{mg} / \mathrm{mL}$ BSA.

\subsection{Continuous monitoring of $\mathrm{Ca}^{2+}$ influx in response to stimulation}

The human embryonic kidney cell line HEK293A ${ }^{(20)}$ was maintained in Dulbecco's modified Eagle's medium containing $25 \mathrm{mM}$ glucose (Wako, Osaka, Japan) with 10\% (v/v) heat-inactivated fetal bovine serum (Funakoshi, Tokyo, Japan), 100 units $/ \mathrm{mL}$ penicillin, and $100 \mu \mathrm{g} / \mathrm{mL}$ streptomycin (Thermo Fisher Scientific) in $5 \% \mathrm{CO}_{2}$ atmosphere at $37{ }^{\circ} \mathrm{C}$. The HEK293A cells were transfected with the plasmid constructed as described in Sect. 2.1 using Lipofectamine LTX (Thermo Fisher Scientific) following the manufacturer's protocol. Transfected cells were cultivated and selected with $500 \mu \mathrm{g} / \mathrm{mL}$ G418 (Nacalai Tesque) for 
approximately two weeks. For the continuous monitoring of $\mathrm{Ca}^{2+}$ influx, transfected cells were collected and inoculated in a 96-well microplate (Thermo Fisher Scientific) at $1 \times 10^{4}$ cells/ well. The following day, cells were washed and incubated for $30 \mathrm{~min}$ in Krebs-Ringer buffer (1.5 mM CaCl $2,0.5 \mathrm{mM} \mathrm{MgCl}_{2}, 4.5 \mathrm{mM} \mathrm{KCl}, 120 \mathrm{mM} \mathrm{NaCl}, 0.7 \mathrm{mM} \mathrm{Na}_{2} \mathrm{HPO}_{4}, 1.5 \mathrm{mM}$ $\mathrm{NaH}_{2} \mathrm{PO}_{4}, 15 \mathrm{mM} \mathrm{NaHCO}$, and $\mathrm{pH}$ 7.4) containing $10 \mathrm{mM}$ glucose and $1 \mathrm{mg} / \mathrm{mL}$ BSA. Cells were further cultured in $49 \mu \mathrm{L}$ of fresh Krebs-Ringer buffer with $10 \mathrm{mM}$ glucose and $1 \mathrm{mg} / \mathrm{mL}$ BSA after washing with the same Krebs-Ringer buffer. Nano-Glo Luciferase Assay Substrate $(1 \mu \mathrm{L})$ was added to the Krebs-Ringer buffer, followed by the addition of Krebs-Ringer buffer containing cell-stimulating factors, namely, $\mathrm{KCl}(50 \mathrm{mM}$ final) and arginine $(15 \mathrm{mM}$ final; Wako). Cellular luminescence was measured using a luminometer (Phelios, ATTO) at 300-600 $\mathrm{nm}$ luminescence for NLuc and $600-650 \mathrm{~nm}$ luminescence for mScarletI with a $3 \mathrm{~s}$ integration time setting. BRET signals were calculated as described in Sect. 2.3.

\section{Results and Discussion}

\subsection{Screening of novel luminescent and fluorescent protein pair for BRET analysis}

To evaluate the novel use of luminescent and fluorescent protein pairs for BRET analysis, the luminescent and fluorescent fused proteins were genetically generated. In this study, we selected mCherry ${ }^{(11,21,22)}$ and $\mathrm{mScarlet}^{(16)}$ as the red fluorescent proteins. The BRET efficiency is dependent on the emission wavelength of the luminescent protein and the excitation wavelength of the fluorescent protein. As the two wavelengths move closer to each other, the BRET efficiency becomes higher; however, the $\mathrm{S} / \mathrm{N}$ ratio becomes lower. Therefore, we selected NLuc ${ }^{(13,14)}$ and ELuc ${ }^{(15)}$ as the blue and green luminescent proteins, respectively, which have separate peak wavelengths from the red fluorescent protein. The genetically fused luminescent proteins (ELuc or NLuc) and fluorescent proteins (mCherry or mScarletI) connected by a linker peptide were fabricated, and the luminescence spectra of the fused proteins were examined. In the case of using both fused proteins with mCherry (mCherryELuc, mCherry-NLuc), only low intensities of BRET signals were detected [Figs. 1(a) and 1(b)]. However, both proteins with mScarletI (mScarletI-ELuc, mScarletI-NLuc) showed high intensities of BRET signals [Figs. 1(c) and 1(d)]. Specifically, the fused protein with mScarletI and ELuc showed a higher BRET signal intensity than the previously reported red fluorescent protein. ${ }^{(8,11,12)}$ Furthermore, mScarletI-NLuc showed a peak distance wavelength that is between the emission of NLuc and the excitation of mScarletI. It indicates that BRET analysis at high $\mathrm{S} / \mathrm{N}$ ratios can be performed using the fused protein pair (mScarletI-NLuc) [Fig. 1(d)]. Therefore, the pair of mScarletI and NLuc could be suitable as a protein probe for BRET-based analysis. In particular, if the luminescent and fluorescent protein pair of mScarletI and NLuc could be designed and used as biosensing probes, it could be applied to the measurement of cell cultures and tissues in living organisms. 


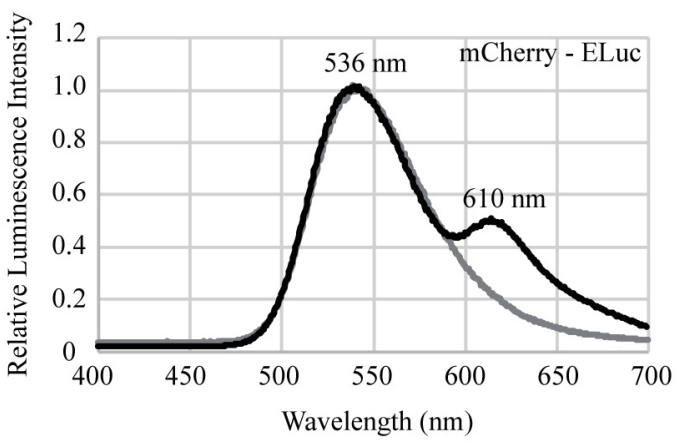

(a)

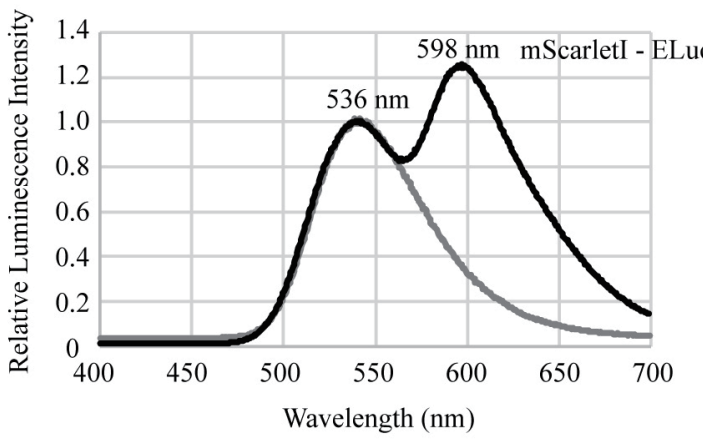

(c)

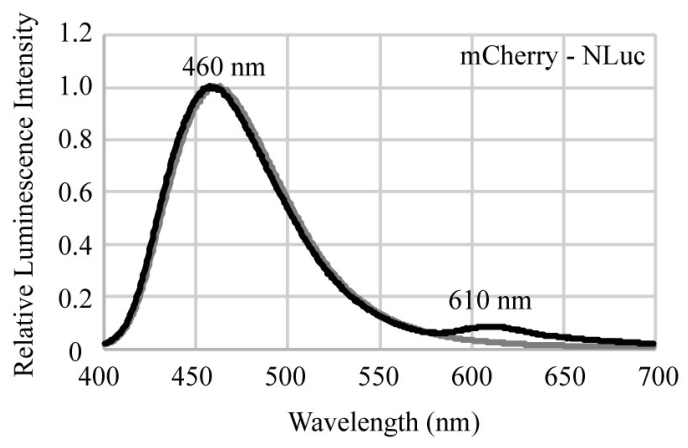

(b)

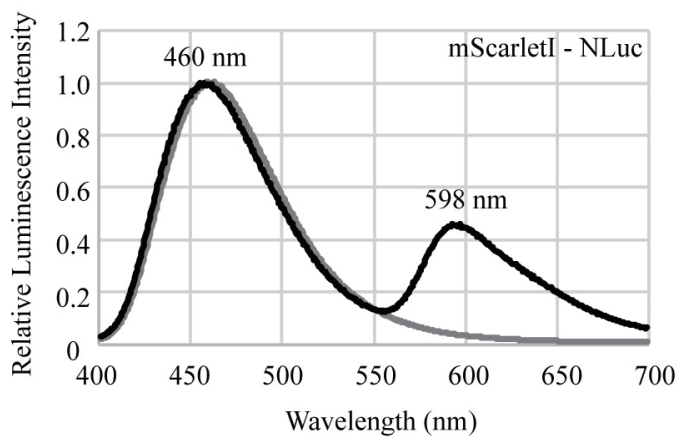

(d)

Fig. 1. Luminescence spectra of the luminescent and fluorescent fused proteins. The black lines indicate the spectra of the fused proteins. The gray lines indicate the luminescence spectra of the original luminescent proteins (ELuc, NLuc). (a) mCherry-ELuc. (b) mCherry-NLuc. (c) mScarletI-ELuc. (d) mScarletI-NLuc.

\subsection{Evaluation of $\mathrm{Ca}^{2+}$-sensing protein probe with novel protein pair}

The novel luminescent and fluorescent protein pair, mScarletI and NLuc, was used in the BRET-based analysis in this study. As a $\mathrm{Ca}^{2+}$ sensing method, Miyawaki et al. developed the FRET-based $\mathrm{Ca}^{2+}$ sensor, cameleon, which is composed of a cyan fluorescent protein (CFP), calmodulin $(\mathrm{CaM})$, a glycine-glycine linker, the CaM-binding peptide of myosin light-chain kinase (M13), and a yellow fluorescent protein (YFP) with a linear combination. ${ }^{(16-18)}$ The target recognition site of $\mathrm{Ca}^{2+}$ was fused with the NLuc protein as the donor and the mScarletI protein as the acceptor. The luminescence spectrum of the protein that reacted with $\mathrm{Ca}^{2+}$ was measured using a BRET-based $\mathrm{Ca}^{2+}$ sensor probe with mScarletI and NLuc [Fig. 2(a)]. Although the peak intensity slightly shifted to the long-wavelength side depending on the $\mathrm{Ca}^{2+}$ concentration, the peak of luminescence spectra was increased [Fig. 2(a)]. Furthermore, the luminescence intensity of mScarletI increased depending on the $\mathrm{Ca}^{2+}$ concentration $(0-4 \mu \mathrm{M})$ [Fig. 2(b)]. These results suggested that the conformation of the fusion protein was changed, and mScarletI and NLuc became close to each other after binding to $\mathrm{Ca}^{2+}$. Therefore, this fusion protein with mScarletI and NLuc could function as a $\mathrm{Ca}^{2+}$-sensing protein probe. The developed protein probe showed the same sensitivity level as the Kd value (14.2 $\mathrm{nM}$ ) compared 


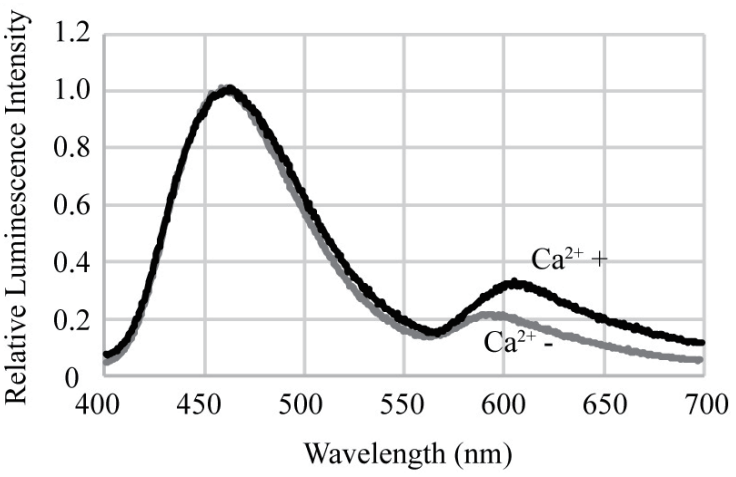

(a)

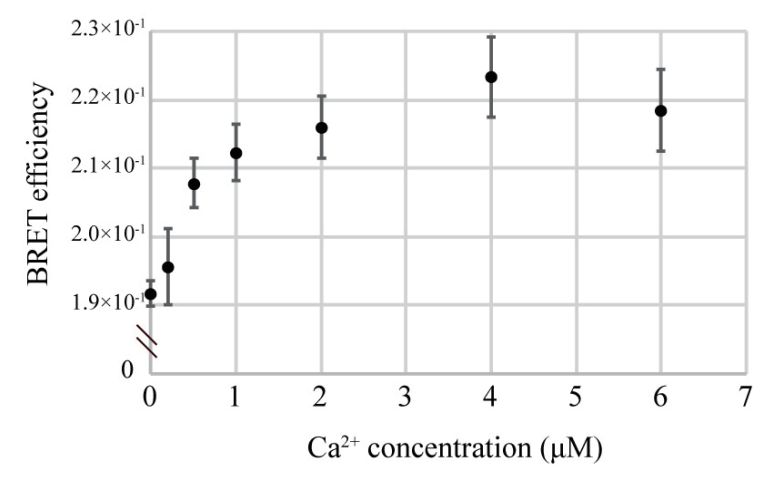

(b)

Fig. 2. Functional analysis of the $\mathrm{Ca}^{2+}$-sensing protein probe with novel luminescent and fluorescent protein pair (mScarletI and NLuc). (a) Luminescence spectra of $\mathrm{Ca}^{2+}$ fusion protein with and without $\mathrm{Ca}^{2+}$. The spectrum indicated by the black line shows that the protein reacted with $4 \mu \mathrm{M} \mathrm{Ca}^{2+}$. The spectrum indicated by the gray line corresponds to the luminescencet protein without $\mathrm{Ca}^{2+}$. (b) Dose response of the BRET-based homogeneous $\mathrm{Ca}^{2+}$ assay. The results in the figures are shown as mean of three replicates \pm standard deviation.

with the previously reported $\mathrm{Ca}^{2+}$-sensing protein probe ${ }^{(17)}$. The limit of detection (LOD) was $0.2 \mu \mathrm{M}$, and the standard deviation was defined to be 3.29 times higher than the average of the blank value $\left(0 \mu \mathrm{M} \mathrm{Ca}^{2+}\right)$. ${ }^{(23)}$

The changes in the intracellular $\mathrm{Ca}^{2+}$ concentration were evaluated using the protein probe expressed in the living HEK293 $\mathrm{A}^{(20)}$ cell line. $\mathrm{KCl}^{(24-26)}$ and arginine ${ }^{(27)}$ were used in the cell assay to stimulate the influx of $\mathrm{Ca}^{2+}$ into the intracellular cytosol. The luminescent substrate was added to HEK293A cells expressing the protein probe, and the cells were stimulated using $50 \mathrm{mM} \mathrm{KCl}$ or $15 \mathrm{mM}$ arginine (Fig. 3). Immediately thereafter, the luminescence signal emitted from NLuc and the fluorescence signal emitted from mScarletI were continuously monitored. The BRET signal intensity of the protein probes representing the $\mathrm{Ca}^{2+}$ concentration in HEK293A cells increased immediately after stimulation with $\mathrm{KCl}$ or arginine solution (Fig. 3). In contrast, HEK293A cells without stimulation did not show an increase in the BRET signal intensity. These results indicate that the $\mathrm{Ca}^{2+}$ influx into HEK293A cells resulting from stimulation was successfully monitored by BRET-based assays using the new protein probe. Although the increase in the BRET signal intensity in HEK293 cells was observed, as shown in Fig. 3, these signal intensities were lower than those of only the $\mathrm{Ca}^{2+}$ solution, as shown in Fig. 2. It is considered that intracellular $\mathrm{Ca}^{2+}$ influx might be interfered by other intracellular molecules. We are planning to investigate and improve the optimum conditions of the biosensing probes in the future. The luminescence was stable in the observation for more than $1 \mathrm{~h}$ in every experiment. The responses of $\mathrm{Ca}^{2+}$ influx observed using the proposed protein probe were consistent with previously reported behavior. ${ }^{(24-27)}$ Therefore, a BRET-based protein probe for cellular imaging can be developed using the novel luminescent and fluorescent protein pair, mScarletI and NLuc. The protein probes using mScarletI and NLuc with high BRET efficiency are expected to be useful for BRET-based imaging and the monitoring of various cells and tissues as these methods will be improved in the future. 


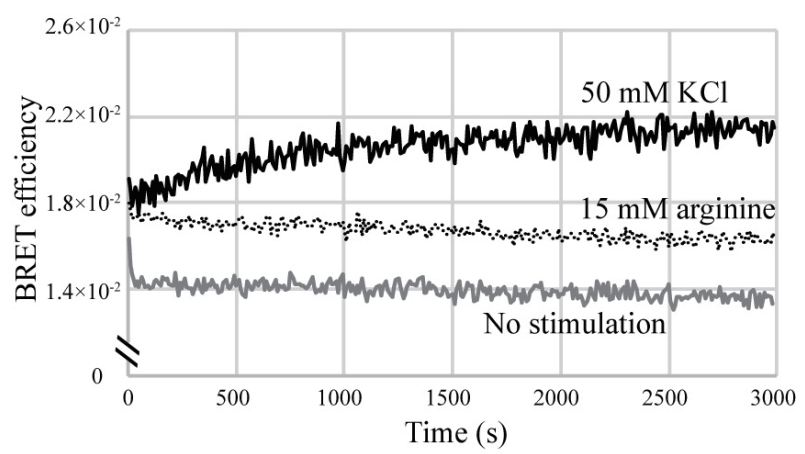

Fig. 3. Continuous monitoring of intracellular $\mathrm{Ca}^{2+}$ concentration in stimulated HEK293A cells by measuring BRET signals. The black line indicates the BRET efficiency upon stimulation with $50 \mathrm{mM} \mathrm{KCl}$. The black dotted line indicates the BRET efficiency upon stimulation with $15 \mathrm{mM}$ arginine and the gray line indicates the control BRET efficiency without stimulation.

\section{Conclusions}

In this study, we screened novel luminescence and fluorescence protein pairs for BRET analysis. The mScarletI and NLuc protein pair showed BRET signals with a high $\mathrm{S} / \mathrm{N}$ ratio and efficient energy transfer. Furthermore, the mScarletI and NLuc protein pair was found to be applicable to the BRET-based protein probe for sensing $\mathrm{Ca}^{2+}$. The BRET-based protein probe with the new protein pair showed that BRET signal intensity is dependent on $\mathrm{Ca}^{2+}$ concentration and enabled the successful identication of intracellular $\mathrm{Ca}^{2+}$ influxes in HEK293A cells stimulated with $\mathrm{KCl}$ and arginine. Therefore, this novel protein pair can be used to construct various BRET-based protein probes for sensing cell and tissue samples.

\section{Acknowledgments}

This work was supported by JSPS KAKENHI Grant Numbers 17H07400 and 18K15299, and JST CREST Grant Number JPMJCR15G4. We thank Dr. Yoshihiro Nakajima (National Institute of Advanced Industrial Science and Technology) for the kind donation of HEK293A cells and assistance with the luminescence assays.

\section{References}

1 D. V. Smirnova and N. N. Ugarova: Photochem. Photobiol. 93 (2017) 436.

2 S. Roura, C. Gálvez-Montón, and A. Bayes-Genis: J. Cell. Mol. Med. 17 (2013) 693.

3 M. Yasunaga, K. Murotomi, H. Abe, T. Yamazaki, S. Nishii, T. Ohbayashi, M. Oshimura, T. Noguchi, K. Niwa, Y. Ohmiya, and Y. Nakajima: J. Biotechnol. 194 (2015) 115.

4 H. Shigeto, T. Ikeda, A. Kuroda, and H. Funabashi: Anal. Chem. 87 (2015) 2764.

5 K. Saito, N. Hatsugai, K. Horikawa, K. Kobayashi, T. Matsu-Ura, K. Mikoshiba, and T. Nagai: PLoS One 5 (2010) e9935. https://doi.org/10.1371/journal.pone.0009935

6 A. Takai, M. Nakano, K. Saito, R. Haruno, T. M. Watanabe, T. Ohyanagi, T. Jin, Y. Okada, and T. Nagai: Proc. Natl. Acad. Sci. 112 (2015) 4352. 
7 M. J. Lohse, S. Nuber, and C. Hoffmann: Pharmacol. Rev. 64 (2012) 299.

8 A. De, A. Jasani, R. Arora, and S. S. Gambhir: Front. Endocrinol. (Lausanne) 4 (2013) 1.

9 P. G. Wu and L. Brand: Anal. Biochem. 218 (1994) 1.

10 T. C. Doyle, S. M. Burns, and C. H. Contag: Cell. Microbiol. 6 (2004) 303.

11 G. Borghei and E. A. H. Hall: Analyst 139 (2014) 4185.

12 J. Chu, Y. Oh, A. Sens, N. Ataie, H. Dana, J. J. Macklin, T. Laviv, E. S. Welf, K. M. Dean, F. Zhang, B. B. Kim, C. T. Tang, M. Hu, M. A. Baird, M. W. Davidson, M. A. Kay, R. Fiolka, R. Yasuda, D. S. Kim, H. L. Ng, and M. Z. Lin: Nat. Biotechnol. 34 (2016) 760.

13 M. P. Hall, J. Unch, B. F. Binkowski, M. P. Valley, B. L. Butler, M. G. Wood, P. Otto, K. Zimmerman, G. Vidugiris, T. MacHleidt, M. B. Robers, H. A. Benink, C. T. Eggers, M. R. Slater, P. L. Meisenheimer, D. H. Klaubert, F. Fan, L. P. Encell, and K. V. Wood: ACS Chem. Biol. 7 (2012) 1848.

14 C. G. England, E. B. Ehlerding, and W. Cai: Bioconjugate Chem. 27 (2016) 1175.

15 Y. Nakajima, T. Yamazaki, S. Nishii, T. Noguchi, H. Hoshino, K. Niwa, V. R. Viviani, and Y. Ohmiya: PLoS One 5 (2010) e10011. https://doi.org/10.1371/journal.pone.0010011

16 D. S. Bindels, L. Haarbosch, L. van Weeren, M. Postma, K. E. Wiese, M. Mastop, S. Aumonier, G. Gotthard, A. Royant, M. A. Hink, and T. W. J. Gadella, Jr: Nat. Methods 14 (2016) 53.

17 A. Miyawaki, J. Llopis, R. Heim, and J. McCaffery: Nature 388 (1997) 882.

18 K. Truong, A. Sawano, H. Mizuno, H. Hama, K. I. Tong, T. K. Mal, A. Miyawaki, and M. Ikura: Nat. Struct. Biol. 8 (2001) 1069.

19 K. Horikawa, Y. Yamada, T. Matsuda, K. Kobayashi, M. Hashimoto, T. Matsu-Ura, A. Miyawaki, T. Michikawa, K. Mikoshiba, and T. Nagai: Nat. Methods 7 (2010) 729.

20 M. Hill, N. W. Iaa, F. Graham, R. Smiley, W. C. Russell, and R. Nairn: J. Gen. Virol. 36 (1977) 59.

21 L. Albertazzi, D. Arosio, L. Marchetti, F. Ricci, and F. Beltram: Photochem. Photobiol. 85 (2009) 287.

22 B. Looyenga, C. VanOpstall, Z. Lee, J. Bell, E. Lodge, K. Wrobel, E. Arnoys, and L. Louters: Sci. Rep. 6 (2016) 29130.

23 L. A. Currie: Pure Appl. Chem. 67 (1995) 1699.

24 N. Dionisio, T. Smani, G. E. Woodard, A. Castellano, G. M. Salido, and J. A. Rosado: Biochim. Biophys. Acta, Mol. Cell. Res. 1853 (2015) 1145.

25 T. Kim, J. Choi, S. Kim, O. Kwon, S. Y. Nah, Y. S. Han, and H. Rhim: Biochem. Biophys. Res. Commun. 324 (2004) 401.

26 J. Follett, B. Darlow, M. B. Wong, J. Goodwin, and D. L. Pountney: Neurotoxic Res. 23 (2013) 378.

27 E. Eroglu, S. Hallström, H. Bischof, M. Opelt, K. Schmidt, B. Mayer, M. Waldeck-Weiermair, W. F. Graier, and R. Malli: Nitric Oxide, Biol. Chem. 70 (2017) 59. 